\title{
Ligadura de Hipogástricas en Manejo de Hemorragias Ginecobstétricas
}

\author{
INSTITUTO MATERNO INFANTIL DE BOGOTA \\ HOSPITAL REGIONAL SAN RAFAEL \\ DE FACATATIVA
}

Dres.: Pío Iván Gómez Sánchez*, Hernando Gaitán Duarte**

\section{INTRODUCCION}

En nuestra práctica diaria es frecuente encontrarnos con casos de hemorragia, ya sea posterior a un parto o a una intervención ginecológica que no cede con el manejo convencional específico para cada caso; la hemorragia post-parto es una patología vista a diario en todas las Instituciones y quizás en nuestra Institución sea más frecuente si tenemos en cuenta que el Instituto Materno Infantil de Bogotá (IMI) atiende en su gran mayoría embarazos de alto riesgo. A diario vemos también que no es infrecuente encontrar que este sangrado post-parto no ceda al uso de ocitócicos, masaje uterino, revisión uterina e incluso taponamiento uterino, quedando como única opción en nuestro medio la posibilidad quirúrgica ya que no contamos con posibilidades de hacer embolización selectiva por angiografía, como tampoco con prostaglandinas parenterales ni mucho menos con trajes neumáticos, como el pantalón antichoque militar que encontramos en la literatura como medidas eficaces en el control de la hemorragia pélvica. Si tenemos en cuenta que gran parte de las pacientes afectadas por esta patología son pacientes jóvenes y con baja paridad no podemos tener como única opción quirúrgica la histerectomía,

Instructor Asociado, Departamento de Ginecología y Obstetricia, Universidad Nacional de Colombia, Instituto Materno Infantil. Jefe de Ginecología, Hospital Regional San Rafael de Facatativá.

** Instructor Asociado, Departamento de Ginecología y Obstetricia, Universidad Nacional de Colombia, Instituto Materno Infantil. por lo cual en estas pacientes preconizamos cirugías menos agresivas como son la ligadura selectiva de Uterinas, Ilíacas Internas y/u ováricas, con el fin de preservar la capacidad reproductora de la mujer. La ligadura de ilíacas internas o hipogástricas tiene múltiples usos como en embarazos abdominales con sangrados pélvicos incontrolables, en sangrados cervicovaginales incontrolables como en el caso de carcinoma de cervix sangrantes donde técnicamente se hace muy difícil una histerectomía, en manejo de hematomas en expansión vaginales, retroperitoneales, de ligamentos anchos y en sangrados de cúpula vaginal posterior a histerectomía. Debemos insistir en que es un procedimiento rápido y sencillo teniendo conocimiento de la anatomía pertinente.

\section{REVISION HISTORICA}

La primera ligadura de hipogástricas fue realizada por Stephens en 1812 como tratamiento de un aneurisma de arteria glútea (25).

El primer uso ginecológico de ligadura hipogástrica fue descrito por el Dr. Howard Kelly del Hospital Johns Hopkins en marzo de 1894, siendo realizada esta intervención para el manejo del sangrado después de fallar al intentar una histerectomía por carcinoma de cervix (15). En 1896 W.R. Pryor, profesor del Hospital Policlínico de New York, recomendaba la ligadura de hipogástricas profilácticamente antes de histerectomía para patología uterina maligna (15). Hecht y Blumenthal en 1954 describen esta técnica para sangrado de cúpula vaginal posterior a histerectomía total abdominal (15). 
Konisberg reporta una serie de 45 casos recolectados hasta 1907 (25).

Kirschbaum en 1950 reporta dos casos exitosos de ligadura de hipogástricas, el primero de un ectópico con sangrado incontrolable del ligamento ancho y el segundo caso, en un post-operatorio de histerectomía abdominal por miomatosis (25).

Tajes en 1956 reporta dos ligaduras como medida definitiva para reducir y controlar el sangrado durante el estado abdominal de una resección abdominoperineal para carcinoma rectal. El mismo autor reporta áreas pequeñas de necrosis en glúteos, posterior a ligadura de hipogástricas en zonas correspondientes a distribución periférica de glúteos superior e inferior (25).

Defletsen en 1956, citado por Sagarra (25), reporta uso de ligadura de hipogástricas para hemorragias uterinas incontrolables.

Ubelhor y Canónico en 1957 citados por Sagarra (25), reportan usos de la técnica en casos urológicos y en cirugía para cáncer rectal respectivamente.

\section{Anatomía}

Es muy importante tener conocimientos claros de la anatomía retroperitoneal para evitar complicaciones y hacer más rápidamente el procedimiento.

Recordemos que todas las estructuras que se deben tener en cuenta en este procedimiento son retroperitoneales; la aorta a nivel de la cuarta vértebra lumbar se bifurca en las arterias ilíacas comunes, que a su vez se dividen en arterias ilíacas externas e internas (hipogástricas). La ilíaca externa discurre a lo largo del músculo psoas ilíaco, lateral y ventralmente al muslo donde se convierte en arteria femoral. La arteria hipogástrica comienza en la bifurcación de la ilíaca común y se extiende bajo el borde superior del gran foramen sacrocíatico, transcurre inferomedialmente a lo largo del borde del psoas ilíaco hacia la pelvis. El promontorio es importante como reparo interno del nivel de la bifurcación de la arteria ilíaca común. La arteria hipogástrica está limitada adelante por el peritoneo, anteriormente el uréter pasa adherido a la cara posterior del peritoneo y cruza la hipogástrica en dirección medial sobre su origen. Posterolateralmente se extiende la vena ilíaca externa y el nervio obturador. Posteromedialmente está la vena ilíaca interna. Lateralmente a la hipogástrica se encuentran los músculoś psoas mayor y menor. Al alcanzar la hipogástrica la pelvis, da dos troncos: anterior y posterior que irrigan las vísceras y paredes de la cavidad pélvica, así como órganos de la reproducción y parte superior e interna del muslo, (Tabla No. 1).

TABLA 1

RAMAS DE ARTERIA HIPOGASTRICA

\begin{tabular}{|l|l|}
\multicolumn{1}{c|}{} & TRONCO \\
\cline { 2 - 3 } \multicolumn{1}{c|}{} & ANTERIOR \\
\hline Ramas Parietales & Ramas Viscerales \\
\hline Obturatrlz & Vesical Superior \\
Glútea Inferior & Vesical Inferior \\
Pudenda interna & Hernorroidal Media \\
& Umbilical \\
& Uterina \\
& Vaginal \\
& Ciática
\end{tabular}

\section{TRONCO POSTERIOR \\ Ramas Parietales \\ Glútea Superior \\ Sacras Laterales}

Ileolumbar

La irrigación de la pelvis, posterior a la ligadura de hipogástricas, está dada por la gran cantidad de anastomosis existentes, las cuales pueden ser homolaterales (verticales) o pueden atravesar la línea media (horizontales), siendo más importantes las verticales en la ligadura bilateral.

Cuando se practica ligadura hipogástrica unilateral la suplencia del lado de la pelvis afectado está dado por la ilíaca interna contralateral y sus ramas al igual que por canales anastomóticos que incluyen:

a. Mesentérica inferior.

b. Epigástrica inferior. 
c. Circunfleja femoral media.

d. Femoris profunda

e. La última lumbar.

f. Sacra media.

g. Circunfleja ilíaca.

Posterior a la ligadura hipogástrica bilateral la suplencia está dada por:

a. Arterias ováricas.

b. Epigástrica inferior (rama de la ilíaca externa).

c. Hemorroidal superior (rama de la mesentérica inferior).

d. Circunfleja y ramas perforantes de la femoral profunda.

e. Lumbares bajas.

\section{Técnica operatoria y complicaciones}

Una vez practicada laparotomía mediana se rechazan las vísceras abdominales con un rollo, mientras un ayudante mantiene si es necesario, el útero hacia adelante, cuando está presente. Posteriormente los pasos a seguir son:

1. Visualización y palpación de estructuras vasculares a través del peritoneo, (ver Figura 1a.).

2. Incisión de peritoneo entre dos pinzas, lateral al uréter y medial al origen de la ilíaca interna (ver Figura 1b.).

3. Disección tejido areolar, rechazando uréter medialmente (ver Figura 1c.).

4. Identificación por palpación de la arteria ilíaca interna y elevación de la misma con pinza de babcock.

5. Disección lateral y medial de la arteria con pinza de ángulo recto (pinza cística).

6. Doble ligadura de la arteria con seda 0 ó 1 separados entre sí aproximadamente $0.5 \mathrm{~cm}$. No cortar, (ver Fig. 1d.).
7. Verificar ausencia de pulso posterior a la ligadura.

8. Peritonización previa revisión de la hemostasia del tejido areolar graso retroperitoneal.

FIGURA 1

\section{LIGADURA HIPOGASTRICAS}

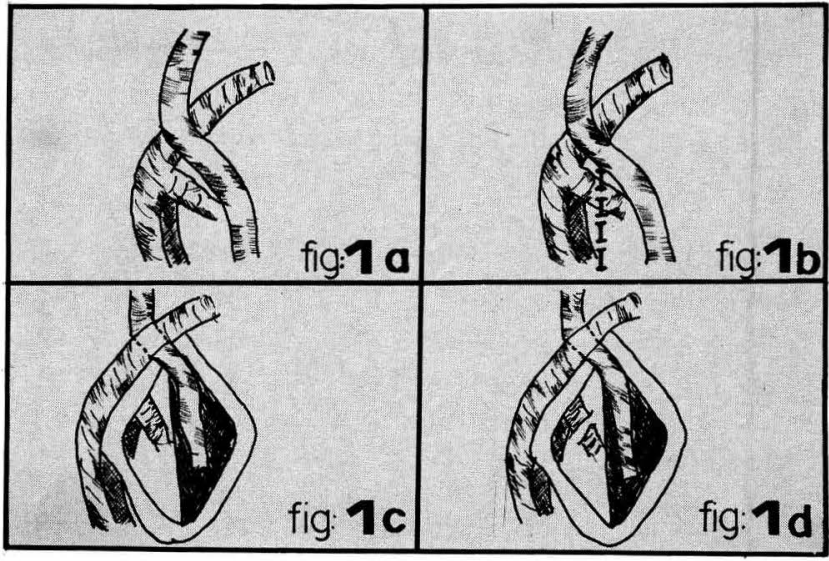

Es de anotar que el cirujano trabaja en el lado opuesto a la arteria. La descripción anterior corresponde a la técnica transperitoneal, utilizada en el IMI. También se conoce la técnica extraperitoneal, que tiene el inconveniente de requerir 2 incisiones y no permitir la revisión de la cavidad pélvica, quedando reservada para casos en que no se requiera dicha exploración como los carcinomas infiltrantes del cervix. Sin embargo, la vía a escoger dependerá de la habilidad del cirujano y su experiencia.

Dentro de la técnica transperitoneal también existen variaciones para la apertura del peritoneo pélvico. La descrita con anterioridad o entrando por las goteras parietocólicas y haciendo mayor disección pues la primera estructura encontrada por esta vía es la arteria ilíaca externa; hay también una descripción reciente del Dr. Cruikshank en 1986, donde se incide el peritoneo existente entre el ligamento redondo y la trompa de Falopio, extendiendo la apertura hasta la pared pélvica lateral. En nuestra experiencia se presenta mayor sangrado del tejido areolar retroperitoneal.

Las complicaciones de la ligadura de la arteria hipogástrica pueden ser intraoperatorias o postoperatorias. Dentro de las intraoperatorias tenemos: la lesión del uréter que se puede obviar con el conocimiento de su anatomía, su localización (adherido al 
peritoneo medial), su vasculatura, su movimiento reptante característico y la sensación de deslizarlo entre los dedos (semejante a la de palpar el conducto deferente del testículo).

La segunda complicación sería la lesión de las venas ilíacas, especialmente la interna por su localización posteromedial a la arteria. Esto se puede evitar si se practica una buena disección de la arteria y se eleva con pinza de babcock aislándola con pinza de ángulo recto. Complicación más grave es la lesión accidental de la arteria ilíaca externa, sea por ligadura o por lasceración, las cuales de no ser corregidas acarrean la pérdida del miembro inferior correspondiente, esta complicación es prevenible con el adecuado conocimiento de la anatomía.

Han sido reportadas en la literatura otras complicaciones como: Necrosis glútea, del periné, de mucosa vesical, parestesia del área glútea, atonía vesical, trastornos circulatorios de extremidad inferior, espasmo y/o trombosis de la arteria ilíaca externa.

Sin embargo, la mayoría de las complicaciones son leves y transitorias. Los casos descritos de necrosis glúteas y viscerales se produjeron cuando estas ligaduras se practicaron en el curso de una excenteración pélvica total luego de radioterapia. En general el concepto es de que aparte de las complicaciones intraoperatorias no existen complicaciones posteriores a la ligadura de la arteria hipogástrica, y pensamos al igual que Reich y Nechtow en 1961, que "La mayor complicación de la ligadura de la arteria hipogástrica es esperar demasiado para realizarla" (24). Cruishank en 1986 reporta 110 ligaduras hipogástricas realizadas en la Universidad de West Virginia y en ninguna se encontró alteración vascular en vísceras pélvicas, vejiga, intestino, regiones glúteas o periné (7).

Siempre hubo dudas en cuanto a la posibilidad de embarazos después de la ligadura de hipogástricas, pero reportes de la literatura mundial nos muestran que la capacidad reproductiva de la mujer no se altera, ni siquiera en los casos en que se ligan también las arterias ováricas. Given en 1961 reporta un embarazo posterior a la ligadura bilateral de hipogástricas, Mengert, en 1969 reporta 5 embarazos y 3 de éstos tenían además ligadura de las arterias ováricas.

\section{Mecanismos de acción}

Antes se pensaba que el mecanismo por el cual la ligadura de hipogástricas controlaba la hemorragia, era por detener el flujo sanguíneo en la porción distal a la ligadura. En 1961, Reich y Nechtow encontraron circulación colateral adecuada en 82 pacientes sometidas a esta intervención (24).

Burchell en 1964 demostró el mecanismo verdadero por el cual la ligadura bilateral de hipogástricas controla la hemorragia. Encontró que si seccionamos la arteria uterina después de haber ligado las hipogástricas, la sangre continúa de tal manera que se requiere una ligadura adicional. Burchell haciendo estudios hemodinámicos y por aortogramas demostró que había una eliminación casi total de la presión de pulso en la porción distal a la ligadura, con una disminución del $77 \%$ de la línea basal en el lado de la ligadura y del $85 \%$ cuando las dos arterias eran ligadas, pero sólo del $14 \%$ en el lado opuesto cuando la ligadura era unilateral $(1,2,3)$. El efecto de la ligadura consiste en cambiar un sistema arterial pulsátil en otro de tipo venoso; al disminuir las pulsaciones arteriales pueden formarse coágulos en la porción distal a la ligadura y permanecer in situ en lugar de ser expulsados por la presión de pulso.

\section{OBJETIVOS}

El propósito del trabajo es evaluar la ligadura de la arteria hipogástrica en la cirugía ginecológica y obstétrica como método alternativo en el tratamiento de la hemorragia.

EI objetivo general es difundir la ligadura de la arteria hipogástrica como técnica rápida segura y sencilla en el tratamiento de la hemorragia ginecológica y obstétrica.

Los objetivos específicos son:

- Cuantificar la mortalidad materna por hemorragia en dicho período y cuantificarla, revisando la técnica quirúrgica.

Analizar las causas de la ligadura de arteria hipogástrica en dicho período.

Analizar las complicaciones intra y postoperatorias.

- Analizar la evolución final de dichas pacientes.

- Revisar la técnica quirúrgica.

- Revisar la literatura. 
MATERIALES Y METODOS

a. Tipo de Estudio: Descriptivo, retrospectivo.

b. Población: Pacientes a quienes se les practicó la ligadura de hipogástricas en el Instituto Materno Infantil (IMI), y Hospital San Rafael de Facatativá entre 1985-1988.

c. Recolección de la Información:

- Técnica. Los datos se obtuvieron de las historias clínicas archivadas en los Departamentos de Estadísticas de los Hospitales en mención.

- Instrumento de recolección: constituido por un formulario precodificado.

- Todas las pacientes fueron intervenidas por el grupo de docentes especialistas o residentes de los hospitales.

\section{RESULTADOS}

Durante este período fueron intervenidas 17 pacientes. De éstas, nueve fueron de causa obstétrica por parto o hemorragia obstétrica incorregible, y 8 cirugía ginecológica, (Tablas Nos. 2 y 3 ).

TABLA 2

\section{LIGADURA BILATERAL DE HIPOGASTRICAS}

\begin{tabular}{llllllllll}
\multicolumn{4}{c}{ CAUSA } & \multicolumn{1}{c}{ OBSTETRICA } & \multicolumn{6}{c}{ GINECOLOGICA } \\
\hline Edad & 85 & 86 & 87 & 88 & 85 & 86 & 87 & 88 \\
$<15$ & - & - & - & - & - & - & - & - \\
$16-20$ & - & - & 1 & 1 & - & - & - & - \\
$21-25$ & - & 1 & - & 5 & - & - & 1 & - \\
$26-30$ & - & - & - & 1 & - & - & - & - \\
$31-35$ & - & - & - & - & - & - & - & - \\
$>35$ & - & - & - & - & & 1 & - & 1 & 5 \\
\hline
\end{tabular}

El $88.9 \%$ de las pacientes tenían 2 hijos o menos.

La principal causa de ligadura fue la atonía uterina, seguida del sangrado de cúpula posthisterectomía. Los casos de prevención de sangrado fueron en histerectomía por miomas intraligamentarios técnicamente difíciles de realizar, todos en
TABLA 3

LIGADURA BILATERAL HIPOGASTRICAS

C. OBSTETRICA

\begin{tabular}{ccccc}
\hline Paridad & 85 & 86 & 87 & 88 \\
PI & - & - & - & 3 \\
P2 & - & - & 1 & 4 \\
P3 & - & 1 & - & - \\
P4 & - & - & - & - \\
\hline
\end{tabular}

1988 al mejorar nuestra experiencia. (Tabla No. 4). Las complicaciones se ven en la Tabla No. 5.

TABLA 4

LIGADURA BILATERAL ARTERIAS HIPOGASTRICAS

CAUSA SANGRADO No.

Atonía Uterina

Inversión Uterina

Desgarro Cesárea

Hematomas

L. Ancho.

$1^{8}$

Acretismo

I

Embarazo Abdominal

Sangrado de Cúpula

Sangrado Cervicovaginal

Ca de Cervix

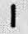

Hemorragia Intraoperatoria

1

Profilaxis Sangrado

Embarazo Ectópico

I

APosthisterectomia abdominal: I Vaginal: 2.

*Paciente con inversión uterinay atonia.

"Pacientes con miomatosis.

- Paciente con desgarro en cesárea y hematoma ligamento ancho. 
TABLA 5

\section{LIGADURA ARTERIAS HIPOGASTRICAS}

\section{TIPO}

Ninguna

14

Lesión arteria hipogástrica

I

Lesión vena ilíaca común (muerte) I

No derivadas de la $Q \times$ (hemotórax)

La paciente que murió fue la primera paciente a la cual se le practicó esta cirugía en 1985. El hemotórax fue causado por punción de vasos subclavios al intentar practicar la cateterización de la vena subclavia para reanimación por shock hipovolémico. El desgarro de la arteria fue parcial y se controló fácilmente intraoperatoriamente. La paciente que sufrió lesión de la arteria hipogástrica durante su disección presentó sangrado, el cual fue controlado rápidamente y se practicó la ligadura. Posteriormente a las 24 horas presentó espasmo arterial de la arteria ilíaca externa que cedió a las 18 horas. La evolución final de las pacientes se ve en la Tabla 6.

TABLA 6

LIGADURA BILATERAL DE HIPOGASTRICAS

\section{EVOLUCION FINAL DE LOS PACIENTES}

\begin{tabular}{cc} 
Salida por mejoría & 14 \\
Muerte-Sepsis obstétrica & 1 \\
- Hemorragia intraop & 1 \\
Reintervención & 1 \\
\hline
\end{tabular}

La paciente que falleció por sepsis obstétrica presentó como patología de base un aborto séptico complicado con peritonitis, practicándosele histerectomía por miometritis, presentando sangrado de cúpula al tercer día P. O. por lo cual se practica la ligadura ya que dicha hemorragia no cedió a medidas conservadoras. Esta paciente presentó además espasmo de la arteria ilíaca externa ipsilateral que cedió espontáneamente.
La paciente reintervenida presentó un severo desgarro cervicovaginal por parto intervenido con espátulas. A dicha paciente se le practicaron 2 revisiones uterinas y drenaje de gran hematoma del espacio pararectal antes de la ligadura, hematoma que se reprodujo luego de dicha ligadura por lo que debió nuevamente ser drenadocon mejoría posterior.

La mayoría de las pacientes estuvieron 6 días o menos posterior a la cirugía en el hospital, (Tabla No. 7).

TABLA 7

\section{LIGADURA BILATERAL DE HIPOGASTRICAS}

E S T A N C I A

\begin{tabular}{lll}
\hline 4 & Dias & 5 \\
$5-6$ & Dias & 9 \\
$8-10$ & Días & 1 \\
$11-15$ & Dias & 0 \\
$>16$ & Días & 1 \\
\hline Muerte Intraop. & 1 \\
\hline
\end{tabular}

\section{COMENTARIOS}

Revisando la mortalidad materna en nuestra Institución desde el año de 1983 hasta 1986 encontramos que la hemorragia ocupa el tercer puesto después de la infección y la toxemia. Este tipo de patología debe ser atacada desde el principio de manera agresiva y rápida.

\begin{tabular}{|lrl|}
\hline Año & Mort. & Casos \\
\hline 1983 & $8.7 \%$ & 4 \\
1984 & $7.3 \%$ & 3 \\
1985 & $10.0 \%$ & 2 \\
1986 & $4.5 \%$ & 1 \\
\hline
\end{tabular}

En el estudio de los Drs.: García y Nañes (8), se encontró que 5 de las 7 pacientes que fallecieron de 1983 a 1984 tenían menos de 29 años.

Revisando las histerectomías obstétricas no infecciosas en el IMI desde 1985 a 1987 fueron 37. 
De éstas, 22 fueron realizadas en pacientes menores de 29 años. Así vemos cómo un gran cupo de mujeres jóvenes requieren medidas quirúrgicas que a más de rápidas y sencillas puedan preservar sus genitales internos, no sólo para conservar su fertilidad sino también por las implicaciones psicosociales que su pérdida implica. En el presente trabajo se practicaron todas las ligaduras de hipogástricas de causa obstétrica en mujeres menores de 29 años y 8 de las 9 ligaduras en mujeres menores de 25 años, resultando grandemente favorecidas las mujeres de este grupo de edad.

En dicho estudio de mortalidad se encontró que 4 de las 7 pacientes tenían 2 hijos o menos. En nuestra revisión se encontró que 8 de las 9 pacientes tenían dicha paridad. Un $33 \%$ de nuestras pacientes eran nulíparas contra un $9 \%$ de las que se sometieron a histerectomía de causa obstétrica entre 1985 a 1988 en nuestro Instituto.

En el $88 \%$ de las pacientes obstétricas la ligadura fue suficiente en el control de la hemorragia. La paciente que requirió reintervención presentaba extensos desgarros cervicovaginales, posteriores a parto vaginal intervenido con espátulas. Fue llevada a cirugía luego de ser sometida a tratamiento médico, 2 revisiones uterinas y drenaje de hematoma perineal con la hemostasia pertinente. Requirió nuevamente cirugía vaginal para hemostasia y drenaje de hematoma con mejoría posterior.

Se debe recordar que a veces es necesario ligar adicionalmente las arterias ováricas (6). Además se recomienda que la ligadura se haga distal a la salida de la rama posterior ya que por allí se podría dar flujo retrógrado a partir de las anastomosis iliolumbar-lumbar y sacra-sacramedial (2).

Al mejorar la experiencia en esta cirugía con la práctica se presentarán menos complicaciones, poco frecuentes pero graves.

Se debe familiarizar al personal que maneja estas urgencias en el hospital con esta cirugía, que lejos de resultar en panacea en el control de la hemorragia obstétrica incohercible, puede convertirse en un arma salvadora para la paciente que presenta hemorragia obstétrica y facilita el control de la hemorragia en ginecología en casos de cáncer inoperables o cirugía pélvica complicada.

\section{CONCLUSIONES}

1. La mortalidad materna por hemorragia sigue siendo la tercera causa de muerte después de la sepsis y toxemia.

2. La mortalidad materna por hemorragia en el IMI ha disminuido en los 3 últimos años. Simultáneamente se ha incrementado el uso de la ligadura de hipogástricas.

3. La principal causa de ligadura de hipogástricas correspondió a la atonía uterina (23.5\%). Igual porcentaje $(2.35 \%)$ ocupó la profiláctica en miomatosis uterina de localización intraligamentaria o miomatosis gigantes. El tercer lugar lo ocupó el uso en hemorragias de cúpula vaginal luego de histerectomías abdominales o vaginales $(17.8 \%)$.

4. La ligadura de hipogástricas fue eficaz en el control de la hemorragia en el $88 \%$ de los casos.

5. Las complicaciones intraoperatorias son previsibles con el conocimiento adecuado de la anatomía retroperitoneal, con técnica quirúrgica depurada y disminuirán con la experiencia del operador.

6. Las complicaciones postoperatorias son muy raras y generalmente transitorias.

7. La estancia de las pacientes depende de su patología de base y no se prolonga por la ligadura de hipogástricas.

8. La ligadura de hipogástricas es un método efectivo en el control de las hemorragias ginecobstétricas y debe hacer parte del entrenamiento básico del Ginecobstetra.

\section{RESUMEN}

Presentamos 17 casos de pacientes ginecobstétricas en las cuales se practicó ligadura de hipogástricas de urgencia o electiva, desde 1985 hasta 1988 en 2 instituciones, el Instituto Materno Infantil y Hospital Regional San Rafael de Facatativá. Se hace una revisión de la anatomía pélvica retroperitoneal, los mecanismos hemodinámicos resultantes de la ligadura que influirán sobre el control de la hemorragia. Se revisan sus complicaciones y la literatura sobre el tema. 
Nueve casos fueron obstétricos y ocho ginecológicos; ocupando el primer lugar de los obstétricos la atonía uterina, y de los ginecológicos el uso intraoperatorio profiláctico seguido del uso en sangrado de cúpula vaginal posthisterectomía.

Se demostró efectividad para cohibir hemorragia en el $88 \%$ de los casos y las complicaciones intraoperatorias desaparecieron a medida que aumentó la experiencia de los cirujanos. Las complicaciones postoperatorias fueron transitorias y no aumentaron la estancia de las pacientes.

\section{BIBLIOGRAFIA}

1. BURCHELL R.C. "Internal iliac artery ligation: Hemodinamics”. Obstet. Gynecol. 24: 737. 1964.

2. BURCHELL R.C.; OLSON G. "Internal iliac artery ligation: aortograms”. Am. J. Obstet, Gynecol. 94: 117, 1966.

3. BURCHELL R.C. "Physiology of internal iliac artery ligation". J. Obstet. Gynecol. Br Commow. 75: 642, 1968.

4. CAVANAGH D. "Urgencias Obstétricas". Editorial Salvat. 3: 27, 1984.

5. CRUIKSHANK S.; STOELK E. "Surgycal control of pelvic hemorrhage: Method of bilateral ovarian artery ligation". Am. J. Obstet. Gynecol. 147: 724, 1983.

6. CRUIKSHANK S.; STOELK E. "Surgycal control of pelvic hemorrhage: Bilateral Hipogastric ligation and method of ovarian artery ligation”. South Med. J. 78: 539, 1985.

7. CRUIKSHANK S. "Tratamiento de hemorragias post-parto y pélvicas”. Clínicas Obstétricas y Ginecológicas. 29 (2): 269 , 1986.

8. GARCIA G.; NAÑEZ H. "Mortalidad Materna en el IMI de Bogotá 1983-1984”. Rev. Col. Obstet y Ginec. Vol. 37 (1): $17,1986$.

9. GIVEN F.T. "Pregnancy following bilateral ligation of the internal iliac arteries. Am J. Obstet Gynecol, 89: 1078, 1964.

10. GOMEZ P.I. "Mortalidad materna en el IMI, 1985-1986 (Monografía).

11. GREISS F.C. "Uterine Vascular response to hemorrhage during pregnancy with observations of therapy". Obstet. Gynecol. 53: 594, 1966.

12. HAYASHI R. "Choque hemorrágico en Obstetricia". Clínicas de Perinatología, 14 (4): 725, 1986.

13. HECHT E.L.; BLUMENTHAL, E.D. "Recurrent Bleeding from the vaginal vault following total abdominal hysterectomy". Am. J. Obstet. Gynecol. 67: 195, 1954.

14. HERBERT W. "Complications of the inmediate puerperium". Clin. Obstet, Gynecol. 25 (1): 219, 1982.

15. HERBERT W.;CEFALO R. "Manejo de hemorragia post-parto”. Clin. Obstet. Ginecol. 27 (1): 181, 1984.

16. HESTER J.D. " Postpartum hemorraghe and reevaluation of uterine packing”. Obstet Gynecol. 45: 5, 1975.

17. LE COCQ F. “Internal iliac artery ligation”. Am. J. Obstet. Gynecol. 95: 320, 1966.

18. LEVENTHAL M.L. "Hemorrhage from carcinoma of cervix control by extraperitoneal ligation of hypogastric arteries" Surg. Gynecol. Obstet 67: 102, 1938.
19. LUCAS W.E. "Postpartum hemorrhage". Clin. Obstet. Gynecol. 23 (2): 637, 1980.

20. MARSDEN D.E.; CAVANAGH D. "Choque hemorrágico en la paciente ginecológica”. Clínicas Obstétricas y Ginecológicas. 13 (2): $483,1985$.

21. MENGERT W.F.; BURCHELL R.C. "Pregnancy after bilateral ligation of the internal iliac and ovarian arteries". Obstet. Gynecol. 34: 664, 1969.

22. O' LEARY J.L.; O' LEARY J.A. “Uterine artery ligation for control of post cesarean section hemorrhage". Obstet. Gynecol. 43: 849, 1974.

23. PARENTE J.T.; DUGLI H. "Pelvic Hemostasis: a new technic and pack". Obstet Gynecol. 19: 218, 1962.

24. PAPALOUCAS A.C. "Extraperitoneal ligation of the internal iliac arteries". Int. Surg. 57: 162, 1972.

25. PRYOR W.R. "Surgical anatomy of the internal iliac artery in woman, and more radical operation for malignant disease of uterus". Am. J. Obstet. Gynecol. 33: 801, 1896.

26. REICH W.J.; NECHTOW J.R. "Ligation of internal iliac arteries; a life-saving procedure for uncontrolable gynecologic and obstetric hemorrhage". Journal of International College of Surgerons. 36: 157, 1961.

27. SAGARRA M. "Ligation of the Internal iliac vessels in the control of postpartum hemorrhage”. Obstet. Gynecol. 15: 698, 1960.

28. SIEGEL P.; MENGERT W.F. "Internal iliac artery ligation in Obstetric and Gynecology”. JAMA, 178: 1059, 1961.

29. SHINAGAWA S. "Extraperitoneal ligation of the internal iliac arteries as a life and uterus saving procedure for uncontrollable postpartum hemorrhage." Am. J. Obstet. Gynecol. 88: $130,1964$.

30. SMITH D.C. "Embolization of the hypogastric arteries in control of massive vaginal hemorrhage". Obstet. Gynecol. 49: $317,1977$.

31. TWOMBLY G. "Hemorragia en cirugía geinecológica". Clínicas Obstétricas y Ginecológicas. 16 (2): 135, 1973.

32. VALLE G. "Hypogastric vessels surgery in Obstetric and Gynecology". Obstet Gynecol Surv. 21: 757, 1966.

33. WATSON P. "Postpartum hemorrhage and shock". Clin. Obstet. Gynecol. 23 (4): 985, 1980.

34. WILliAMS. Obstetricia. Capítulo 1. Editorial Salvat, 1986, p: 3, 3a Ed. 